\title{
Solution and Membrane Interaction Dynamics of Mycobacterium tuberculosis Fatty Acyl-CoA Synthetase FadD13
}

Camilla A. K. Lundgren ${ }^{1,2}$, Michael Lerche ${ }^{1,3}$, Charlotta Norling ${ }^{1,4}$ and Martin Högbom ${ }^{1 *}$

${ }^{1}$ Department of Biochemistry and Biophysics, Stockholm University, Stockholm, Sweden.

AUTHOR INFORMATION

\section{Corresponding Author}

*Correspondence should be addressed to Martin Högbom, e-mail: hogbom@dbb.su.se.

Present Addresses

${ }^{2}$ Sir William Dunn School of Pathology, University of Oxford, Oxford, UK. ${ }^{3}$ AstraZeneca AB, Mölndal, Sweden. ${ }^{4}$ Roche Diagnostics Scandinavia AB, Solna, Sweden. 


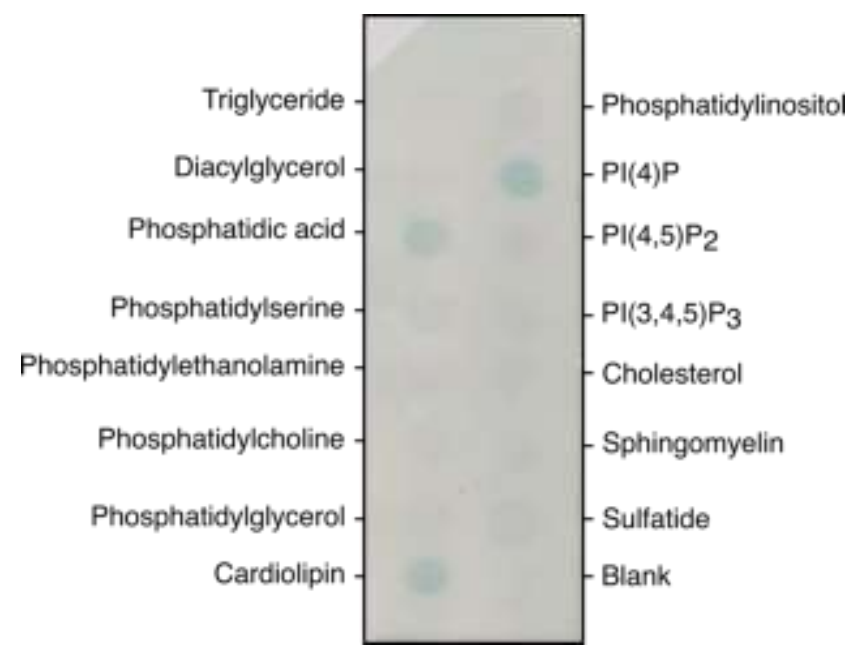

Supplemental figure 1. Lipid strip assay. Representative image of three individual experiments.

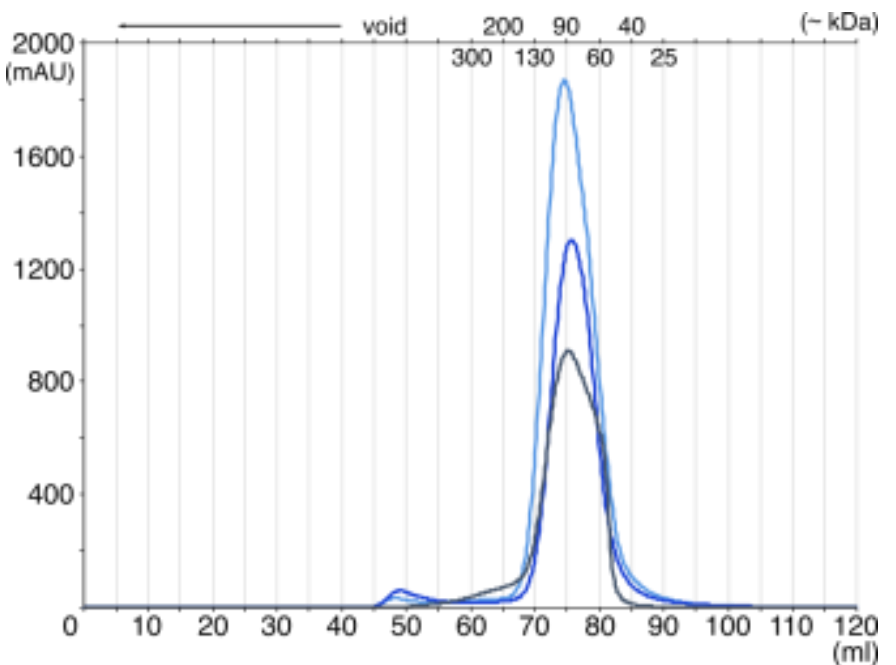

Supplemental figure 2. Gel filtration chromatogram of FadD13. Three individual experiments shown, representative of $\mathrm{n} \geq 10$ experiments. 


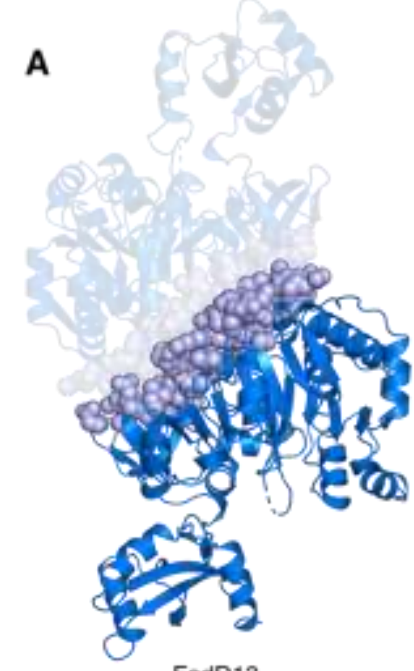

B

FadD13

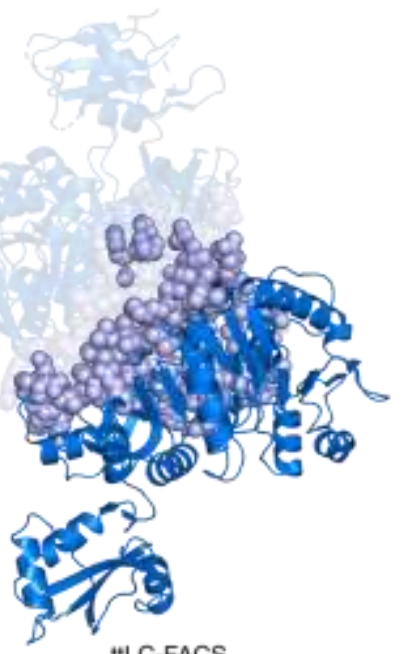

Supplemental figure 3. A comparison between the interface of A) FadD13 dimer (PDB entry 3R44) and B) ttLC-FACS dimer (PDB entry 1ULT). Interface residues highlighted as spheres; one monomer shown as transparent cartoon for clarity.

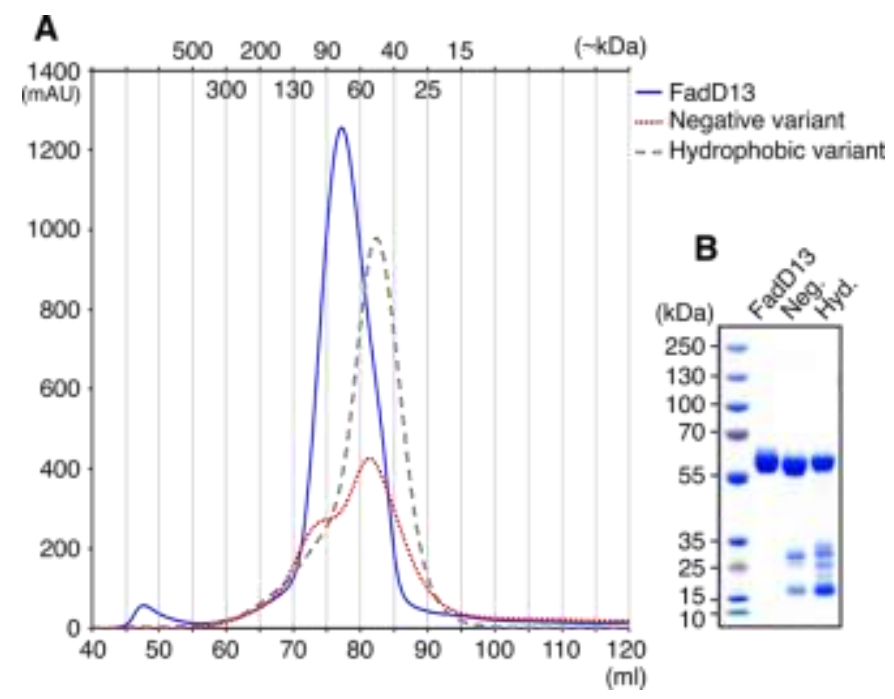

Supplemental figure 4. Purification of FadD13 variants vs. wtFadD13. A) Gel filtration chromatogram of FadD13 (blue, full), negative variant (red, dotted) and hydrophobic variant (gray, broken). B) Coomassie stained SDS-PAGE gel of wtFadD13 and FadD13 variants. 